\title{
HEART RATE VARIABILITY AND FALLS IN HUNTINGTON'S DISEASE
}

Cinthia Terroba-Chambi $\mathrm{MD}^{1,2}$, Veronica Bruno $\mathrm{MD} \mathrm{MPH}^{3}$, Daniel E. Vigo MD, $\mathrm{PhD}^{2,4,5 *}$, Marcelo Merello MD, Ph.D. ${ }^{1,2^{*}}$

\section{AUTHOR AND ARTICLE INFORMATION}

${ }^{1}$ Raul Carrea Institute of Neurological Research, Movement Disorders Unit, FLENI, Buenos Aires, Argentina.

${ }^{2}$ National Scientific and Technological Research Council (CONICET), Buenos Aires, Argentina.

${ }^{3}$ University of Calgary, Department of Neurosciences, Calgary, Alberta, Canada.

${ }^{4}$ Institute for Biomedical Research, Pontifical Catholic University of Argentina (UCA) and National

Scientific and Technological Research Council (CONICET), Buenos Aires, Argentina.

${ }^{5}$ Faculty of Psychology and Educational Sciences, Katholieke Universiteit Leuven, Leuven, Belgium

\section{CORRESPONDING AUTHOR}

Marcelo Merello, MD, Ph.D.; E-mail: mmerello@fleni.org.ar (MM)

* These authors contributed equally to this work

\section{AUTHOR CONTRIBUTIONS}

CTC, VB, DEV, and MM conceptualized and designed the initial project of this manuscript. CTC, VB, $\mathrm{DEV}$, and MM made the final research plan. CTC, VB, DEV, and MM ran the statistical analyses and made a definitive interpretation of the findings. CTC, VB, DEV, and MM wrote the first version of the manuscript. All authors edited and approved the final version of the manuscript

\section{FUNDING:}

CTC is a doctoral research fellow from CONICET. Research award from Fundación HD Lorena Scarafiocca, Buenos Aires-Argentina. The funding agencies did not have a role in the development of the study, analysis of data, and writing of the manuscript.

\section{COMPLIANCE WITH ETHICAL STANDARD}

Conflict of interest: None of the authors have any conflicts of interest to report. 


\section{ABSTRACT}

Purpose: Huntington's disease (HD) patients have a high prevalence of falls. Autonomic nervous system dysfunction has been reported from the early stages of the disease. There is no evidence analyzing the relationship between heart rate variability (HRV) and falls in this population. This research aimed to evaluate the relationship between HRV and falls in HD.

Methods: HD patients enrolled in a prospective study of Fear of Falling and falls were assessed using short-term HRV analyses and blood pressure measures in resting and standing states. Time-frequency domains and nonlinear parameters were calculated. Data regarding falls, the risk of falling (RoF) and disease-specific scales were collected at baseline and six-month follow-up.

Results: Twenty HD patients were recruited. $35 \%$ of HD patients reported at least one fall (single fallers) and, $65 \%$ reported two or more falls (recurrent fallers) in the previous 12-months. At baseline, recurrent fallers had lower RMSSD-resting (root-mean-square-of-the-RR), higher LF/HF ratio (low/high frequency) in both states, and higher DFA- $\alpha 1$ parameter (short-term-detrended-fluctuation-analyses) in both states. This association was similar at a six-month follow-up for recurrent fallers showing lower RMSSD-resting and higher LF/HF-standing ratio than single fallers. Significant correlations were found between the number of falls, RMSSD-resting, and LF/HF-standing ratio. No differences were found between recurrent and single fallers for any blood pressure measures.

Conclusions: The observed HRV pattern is consistent with a higher sympathetic prevalence associated with a higher RoF. Reduced parasympathetic HRV values predict being a recurrent faller at six-months of follow-up, independently of orthostatic phenomena in this population.

Key Words: autonomic nervous system, heart rate variability, fall prediction, risk of falling, Huntington's disease. 


\section{INTRODUCTION}

Huntington's disease (HD) is a neurodegenerative disorder characterized by progressive neural loss affecting mainly the caudate nucleus and putamen within the basal ganglia [1]. Falls are frequent and have a high impact on the quality of life among people with HD [2]. Among people with early to midstage $\mathrm{HD}$, single fall rates range from $21 \%$ to $75 \%$, and recurrent falls range from $58 \%$ to $60 \%$ [3-4], being fall-prone one of the strongest predictors of nursing home placement [5].

Falling in HD is multifactorial in origin. There is a complex interaction between chorea and bradykinesia, and their impact on balance, increasing the chance of falling [4]. Factors such as reduced cognitive reserve for dual-tasking [2], behavioral disturbances such as recklessness, reduced attention, and lack of insight, as well as autonomic [6], may also have an effect. The use of antidepressants, neuroleptics, home environment, cardiovascular medications, and alcohol intake are additional factors that may contribute to falling $[3,7]$. The evaluation of the autonomic nervous system (ANS) as a tool for fall detection is relatively recent. Different studies have used the ANS evaluation and, particularly, heart rate variability (HRV), to detect and predict the risk of falling (RoF) in other populations [6, 8-9]. However, the association between recurrent falls focusing on autonomic dysfunction has not been studied enough in the general population, and there are no previous studies focused on HRV with postural changes in the HD population.

ANS dysfunctions in HD population have been previously identified [10-11]. Most reports showed early sympathetic hyperactivity in the HD population [10, 12-16]. The most likely mechanism underlying these findings is an apoptosis-induced structural defect in the central autonomic network, such as the limbic system, the brainstem, or the hypothalamus [17-18].

Although falls are multifactorial, the evaluation of autonomic dysfunction in HD may be important to investigate its role on RoF to minimize falls and to identify and monitor patients prone to fall [19]. Therefore, the main aim of this study was to investigate the association between the RoF and short-term HRV assessment in different positions in HD.

\section{PATIENTS AND METHODS.}




\section{Design of the study.}

A cross-sectional study was conducted between April 2016 and March 2019 to evaluate the association between clinical and autonomic variables and falls in HD patients. Falls in the previous 12-months and HRV were evaluated at baseline. Additionally, subjects were contacted by phone six months later to obtain information regarding the occurrence of falls in those six-months.

\section{Participants.}

Patients with a genetically confirmed diagnosis of HD enrolled in a study of fear of falling (FoF) and falls were assessed by short-term HRV analysis and blood pressure measures in different positions. Short-term HRV included time-domain, frequency- domain, and nonlinear parameters. These variables were recorded for $5 \mathrm{~min}$ in resting and standing states. Data in each state and the difference between resting and standing states were analyzed. Demographic data including age (years), weight (kg), and current medication was obtained. Disease-specific and validated scales for measuring RoF in HD were performed. Additionally, data regarding falls were collected using a retrospective questionnaire. Participants were divided into two groups: 1 . recurrent fallers if they reported $\geq 2$ falls over the previous 12-months or 2. Single fallers if they reported $\leq 1$ fall [20]. Similarly, the patients were classified based on the occurrence of falls during the six-months of following-up.

\subsection{Inclusion and exclusion criteria}

Participants were eligible for the study if they met the following inclusion criteria: 1) genetically confirmed diagnosis of $\mathrm{HD}$; 2) >18 years of age; 3) stable medication regime for four weeks before assessment. Exclusion criteria included: 1) the presence of diseases known to affect HRV (cardiac arrhythmia, heart failure, arterial hypertension, use of a pacemaker, and kidney or liver disease), 2) diagnosis of dementia based on the DSM-5 criteria, 3) inability to walk independently (use of a cane was allowed).

Whenever possible cardiac medications that could affect the results of HRV (i.e., calcium-channel blockers, mineralocorticoids) were discontinued or reduced to the lowest dosage. Patients with betareceptor blockers treatment were excluded. Angiotensin-converting-enzyme inhibitors, angiotensin receptor blockers, and diuretics were allowed. 
Written informed consent was obtained from all the participants after a detailed explanation of the procedures. The Local Ethics Committee approved the study, which followed all the principles set out in the Declaration of Helsinki.

\section{Baseline assessment}

All tests were performed in a single visit in a standardized order. A neurologist obtained the following clinical measures of disease severity: the Unified Huntington Disease Rating Scale (UHDRS), total motor score (UHDRS-TMS, range 0-124; lower is better), and Total Functional Capacity (UHDRS-TFC, range 0-13; a higher score is better). Following the recommendations of The Kellog International Work Group on the prevention of falls in the elderly, a fall was defined as "an unintentional or unexpected event, it results in the person coming to rest on the ground or another lower level [21]. The history of falls was self-reported and confirmed by a family member or caregiver. Participants were asked to recall if they had any fall in the last 12 months and if the response was positive, to describe the circumstances and any associated injuries.

For a complete assessment of balance and gait, three tests validated for use in HD were performed:

1) The Berg Balance Scale (BBS) [22] a 14-item task common in everyday life surveys related to balance control. Better balance is indicated by higher scores (range 0-56). The BBS is a suggested scale for screening for fall risk [23] with a cutoff score of 40 to predict being a faller [4].

2) The Timed-up go test (TUG) [24] measures in seconds the time taken by an individual to stand up from a standard armchair, walk a distance of three meters, turn, walk back to the chair, and sit down again. The TUG is suggested for assessing the severity of balance and mobility issues, and for screening for fall risk, however, there is no sensitivity or specificity data for the reported cutoff point [23]. Mean scores for patients with manifest HD range from 9 to 17 seconds [25] and the cutoff score of 14 seconds has been reported to predict being a faller [4].

3) The Tinetti mobility test (TMT), a 16-item performance measure (range 0-28) which consists of balance and gait subscales that measure static and dynamic balance [26]. The use of the TMT to screen RoF in HD use, the cutoff value of 21 to distinguish between those who are at high $\mathrm{RoF}(\leq 21)$ from those who are not $(>21)[23,27]$. Additionally, cognitive and neuropsychiatric symptoms were assessed using a comprehensive battery. The battery included the Montreal Cognitive Assessment (MoCA), the Frontal 
Assessment Battery (FAB), the Beck Depression Inventory (BDI), the Beck's Anxiety Inventory (BAI), Starkstein's Apathy Scale (SAS), Fear of Falling Scale (FES-I), and the Barthel Index.

\section{Follow-up assessment}

Participants were contacted by phone to record any fall incidence for six months after the baseline assessment. Participants were asked to complete a falls diary recording every fall experienced during this period, as soon as they occurred. To ensure accurate reporting of falls and to verify check for patients' missing data, their family members and/or caregivers were contacted by phone monthly by the same investigator who recruited and assessed patients at baseline. This method is considered more appropriate to collect fall incidence data [28]. FES-I scale to assess concerns about falling was also completed by phone during the follow-up evaluation.

\section{HRV assessment}

HRV examinations were always performed between 14:00 and 17:00 hrs. To avoid circadian HRV variations. All participants were instructed to have a light meal only, to sleep for at least 7 hours before, and to abstain at least 3 hours from drinking caffeine-containing beverages". Participants were asked to remain in the same position silently during the recording time. The HRV tests were performed in a standardized order, as described below.

\subsection{Signal recording:}

Participants were connected to the ECG-recording device and left resting in a sitting position for $10 \mathrm{~min}$. Then, the ECG signal was recorded for 5 min under the same conditions (resting-state). Afterward, the participants were asked to stand-up, and the ECG was recorded during five additional minutes (standing state). Blood pressure was measured twice under all conditions. Orthostatic hypotension was defined as a sustained reduction of systolic blood pressure of at least $20 \mathrm{mmHg}$ or diastolic blood pressure of 10 $\mathrm{mmHg}$, or both, within $3 \mathrm{~min}$ of standing according to the recommendations of international consensus statement [29]. Electrocardiogram signal was recorded using a digital Holter device (Holter HCAA 348/Holtech/Servicios Computados S.A./Buenos Aires/Argentina) and stored in a memory card. Ventricular depolarizations ( $\mathrm{R}$ waves) were detected through the device software. The Kubios ${ }^{\circledR}$ software 
[30] (Kubios HRV. 2.1, Biosignal Analysis and Medical Imaging Group, Kuopio, Finland) was used to import and analyze all RR series [31-32].

\subsection{HRV analyses}

We analyzed 5-min periods of resting-state and 5-min of standing state RR intervals resulting from sinus beats. Medium threshold correction was used for detecting artifacts (missed, extra, and misaligned beat detections) as well as ectopic beats [30]. The artifacts and ectopic beats were corrected by comparing every RR interval value against a local average interval $(0.25 \mathrm{~ms})$. Detected ectopic beats were corrected by replacing corrupted RR times by interpolated RR values. Missed beats were corrected by adding new R-wave occurrence time, and extra beats were corrected by removing extra R-wave detection and recalculating the RR interval series [30]. All records included in this study had more than $80 \%$ of valid data [31]. For the slow linear or more complex trends within the time-series analyses, the smoothness prior method for removing time series non-stationarities was applied. The strong stationarity was visually checked. The cutoff frequency was below the low-frequency band $(<0.04 \mathrm{~Hz})[30]$.

\subsubsection{HRV linear analyses:}

Time-domain (non-spectral): Time-domain measurement of HRV included the mean HR (HRM), the mean RR interval (RRM, RR interval is defined as the time distance between nearest R peaks in human electrocardiogram), the standard deviation of all regular RR intervals (SDNN), and the root mean square of the successive differences in regular RR intervals (RMSSD) [32]. RRM quantifies the mean heart rate, SDNN represents a coarse quantification of overall variability, and RMSSD measures high-frequency heart rate variations $[17,30,32]$.

Frequency-domain (spectral): In the frequency-domain methods, a spectrum estimate was calculated for the RR interval series. Prior to spectrum estimation, the RR interval series was converted to equidistantly sampled series by cubic spline interpolation. The spectrum was estimated by Welch's periodogram [30]. In Welch's periodogram, the RR series was divided into overlapping segments, each segment was windowed to decrease the leakage effect, and the spectrum estimate was obtained by averaging the Fast Fourier Transform spectra of these windowed segments. The spectrum estimates are then divided into very low frequency (VLF), low frequency (LF), and high frequency (HF) bands. The generally used limits for these bands in the case of short-term HRV recordings in healthy human subjects are $0-0.04 \mathrm{~Hz}$ 
(VLF), 0.04-0.15 Hz (LF) and 0.15-0.4 (HF) [32-34]. HRV measures extracted from these frequency bands included absolute powers, expressed as the natural logarithm for each band $\left(\ln \mathrm{ms}^{2}\right)$ and the LF/HF power ratio [30, 32]. All HRV indices were calculated for resting, standing, and the difference between both conditions.

\subsubsection{HRV nonlinear analyses:}

Scaling exponent $\alpha$ and sample entropy (SampEn) were used as nonlinear HRV indexes. $\alpha$ s, based on the "detrended fluctuation analysis" (DFA), quantifies the short-term fractal correlation properties of the interbeat time data [35]. DFA measures the correlations within the data for different time scales and is divided into short-term and long-term fluctuations, which are characterized by parameters $\alpha 1$ (range 4-16 beats) and $\alpha 2$ (range 16-64 beats), respectively [30]. Values of $\alpha$ close to 0.5 are associated with white noise (no correlation between values), whereas values close to 1.5 are associated with Brownian noise (strong correlation between values). Values near 1 are characteristic of fractal-like processes, associated with the dynamic behavior of time series generated by complex systems, such as the autonomic regulation of the sinus rhythm of a healthy subject. Sample entropy (SampEn) [36] estimated the irregularity of the RR interval time series as a measure of system complexity. Regular sequences will result in lower SampEn values, whereas random behavior is associated with larger SampEn values. These methods have been previously described [34-36]. Uncorrelated and irregular behavior is usually associated with parasympathetic prevalence [37].

\section{Statistical analyses}

The sample size was decided based on the expected differences between risk groups (early-mid/late stages of HD, and faller/no faller) and estimated population SD for selected HRV variable: LF mean difference $\left(1.5 \mathrm{~ms}^{2}\right)$, SD $\left(1.1 \mathrm{~ms}^{2}\right)$; HF mean difference $\left(1.4 \mathrm{~ms}^{2}\right)$, SD $\left(1.0 \mathrm{~ms}^{2}\right)$; LF/HF ratio mean difference $\left(0.4 \mathrm{~ms}^{2}\right), \mathrm{SD}\left(0.3 \mathrm{~ms}^{2}\right)$. Thus, a sample size of 8 participants per group was decided (statistical power of $80 \%$, alpha 0.05 , STATA $13 \mathrm{v}$ ). The values of references used for the calculation have been previously published $[8,15]$. Median and interquartile range $25 \%$ and $75 \%$ or proportions were compared between single and recurrent fallers, through unpaired Mann-Whitney $\mathrm{U}$ tests or $\mathrm{X}^{2}$ tests, respectively. Correlation coefficients between the number of falls and HRV were calculated with Spearman's rho. The statistical significance for these comparisons was set at $\mathrm{p}<0.05)]$. A false discovery rate $(\mathrm{FDR}=0.25)$ 
correction was applied to multiple intergroup comparisons

(https://www.sdmproject.com/utilities/?show5FDR). Logistic regression models were used to assess the independent predictive value of RoF outcome measures and HRV to identify recurrent fallers. Faller status at six-months (single faller / recurrent faller) was included as the dependent variable, while RoF

measured by BBS or TUG or TMT (high risk $=1$, low risk $=0$ ), and significant HRV parameters were included as independent variables. Taking into account the small sample size of our study, models were constructed with only two independent variables as predictors: a clinical scale that measures RoF in HD as the first independent variable [BBS, TUG or TMT], and the HRV variable that had been significant in univariate analysis (RMSSD-resting or LF/HF-standing) as the second independent variable. Thus, each model included two independent predictors that were included as follows: 1. RMSSD-resting and BBS; 2. RMSSD-resting and TUG; 3. RMSSD-resting and TMT; 4. LF/HF-standing and BBS; 5. LF/HF-standing, and TUG; 6. LF/HF-standing and TMT. To discard collinearity between independent variables we used variance inflation factor (VIF), all variables included in logistic regression models had VIF values lower than 10 (data not shown). In addition, we double-checked the absence of collinearity with low VIF measures for all our variables using the command Collin on STATA 13v. Associations were expressed as the coefficient of regression (standard error), and Odds Ratios (OR) [95\% Confidence intervals (CI). Statistical analyses were performed using IBM SPSS Statistics version 23.0 (IBM, Corp, Armonk New York).

\section{RESULTS.}

\section{Demographic and Clinical Characteristics}

Twenty-four HD patients were invited to participate. Three patients refused participation; one patient was excluded because of the use of beta-receptors blockers, leaving a total sample of 20 patients who completed baseline and 18 patients who completed a six-month follow-up. At baseline, there were 12 $(60 \%)$ women. According to UHDRS-TFC $16(80 \%)$, patients were in the early-mid (early-stage, $n=14$; mid-stage, $n=2)$, and $4(20 \%)$ patients in the late stage of the disease. Due to the small number of patients in the mid-stage, this group was unified with the group in the early stage, forming the early-mid group $(\mathrm{n}=16)$. The rationale of forming the early-mid group derives from the clinical similarity in early-stages of the disease with respect to the mid-stage. Medians and interquartile range (25\%-75\%) at baseline of all clinic and demographics variables are presented in Table 1. The BBS classified 7 patients (2 single versus 
5 recurrent fallers, $\mathrm{p}=0.658)$ as high $\mathrm{RoF}(\mathrm{HRF})$, the TUG classified 14 patients $(5$ single versus 9 recurrent fallers, $\mathrm{p}=0.919)$ as HRF, and by the TMT 10 patients $(3$ single versus 7 recurrent fallers, $\mathrm{p}=$ 0.639) were classified as HRF (Table 1).

At baseline, seven patients (35\%) were classified as single fallers and $13(65 \%)$ patients as recurrent fallers (reporting $\geq 2$ falls). In the single fallers group, $3(43 \%)$ reported only one fall, and 4 (57\%) reported no falls. Compared to recurrent fallers, single fallers had a significantly fewer total number of falls in the past 12 months $[\mathrm{U}=91.0, \mathrm{p}<0.001]$, and lower level of FoF by FES-I scores $[\mathrm{U}=71.0, \mathrm{p}=$ 0.043] (Table 1). There were no differences in age, weight, gender, MoCA test, and the remaining clinical and demographic variables between those who were classified as recurrent fallers and those classified as single fallers (Table 1).

At follow-up, the median number of falls was 0.5 (0-2), and the median FES-I was 23.5 (18.5-33). Eleven patients $(61 \%)$ were classified as single fallers, of which $9(82 \%)$ reported no falls, and $2(18 \%)$ reported only one fall. Seven patients (39\%) were categorized as recurrent fallers. As a baseline, significant differences were found in the number of falls between fallers type at follow-up $(U=71.0, p=0.003)$. There were no differences in the FoF level between single and recurrent fallers at follow-up.

\section{Association between HRV, RoF and falls}

At baseline recurrent fallers had significantly lower RMSSD-resting $(\mathrm{p}=0.011)$, higher LF/HF ratio in both states (resting, $\mathrm{p}=0.006$; SS, $\mathrm{p}=0.019$ ) and higher DFA- $\alpha 1$ in both states (resting, $\mathrm{p}=0.024$; standing, $\mathrm{p}=0.006$ ) than single fallers. No significant differences in HRV values of the differences between states were found when comparing patients by groups (single versus recurrent fallers) (Table 2). Similarly, at follow-up recurrent fallers had significantly lower RMSSD-resting $(p=0.027)$ and higher $\mathrm{LF} / \mathrm{HF}$ ratio in standing $(\mathrm{p}=0.035)$ (Table 3). When assessing the association between number of falls and HRV measures the following significant correlations were observed in resting-state between the RMSSD and [number of falls $(r=-0.493, p=0.027)($ Fig. 1A), and LF/HF-standing and number of falls $(\mathrm{r}=0.496, \mathrm{p}=0.026)($ Fig. 1B) (Appendix 1). When RoF based on BBS, TUG, and TMT scores, and HRV measures were assessed, no significant correlations were found in any state (Appendix 3).

\section{Blood pressure measures and falls}


No significant differences were found between recurrent and single fallers for any blood pressure measures (Appendix 2).

\section{Predictive validity of HRV measures}

The results of logistic regression indicated that RMSSD-resting and LF/HF-standing ratio are significantly associated with the odds of being a recurrent faller in the next six months at a level of $\mathrm{p}<$ 0.05. The regression coefficients (standard errors) of RMSSD-resting and LF/HF-standing ratio were $0.084(0.044)$ and $1.580(0.758)$ respectively. The estimated OR $(95 \% \mathrm{CI})$ of RMSSD-resting and LF/HFstanding ratio were $0.917(0.849-0.996, \mathrm{p}<0.036)$, and $4.899(1.099-21.436, \mathrm{p}=0.043)$, respectively. In other words, lower values RMSSD-resting and higher values of LF/HF-standing increase the risk of being a recurrent faller in the next six- months.

\section{Qualitative information about falls}

The most frequent cause and direction of falls were tripping (60\%) and forward (65\%). The place of occurrence of falls most frequently reported was the bathroom during the shower in a stand-up position (30\%). Thirteen (65\%) fallers had injuries by falls; the most frequent injury reported was bruising $(62 \%)$ followed by sprains or dislocations (31\%). Finally, 8 (40\%) fallers required medical assistance for physical damage related to falls.

\section{DISCUSSION}

To our knowledge, this is the first study suggesting a relationship between faller status and short-term HRV in different positions in HD. We have found that patients in early-mid stages of HD do regularly fall (only $20 \%$ of participants did not report any fall in the previous 12-months) and that HRV pattern was consistent with a higher sympathetic prevalence associated with a higher frequency of falls. The decrease of parasympathetic HRV values adequately identifies being a recurrent faller, independently of orthostatic phenomena, in this specific population.

HRV measurement is a complementary non-invasive method commonly used to estimate the ANS activity. $\mathrm{LF} / \mathrm{HF}$ ratio should be interpreted as a measure of relative sympathetic predominance [32, 38]. Other changes in HRV (increased DFA $\alpha 1$, decreased RMSSD) also point in the same direction; therefore, these results might instead point to decreased vagal modulation. Previous studies support these 
results since they showed an underlying structural alteration, the degeneration in central vagal nuclei in HD [39-41].

Melillo et al. [8] showed that a depressed HRV increased the RoF fivefold since the depressed HRV would reflect a reduced ability to react to risk situations [8]. Similarly, Nocua et al. [6] found a positive predictive value of $73.8 \%$ when they included ANS activity to improve the detection rate of fall sensors during simulated falls [6]. In line with our results, other authors [12, 40] identified that the HRV profile differed among early-HD participants who exhibited a lower HF and higher LF variability versus the preclinical groups and controls. Those results suggest a sympathetic predominance in patients with presymptomatic HD and with mild and moderate clinical involvement [41]. In contrast, another study [42] found no differences in the values of LF and HF between the control, pre-symptomatic, and early HD groups. The possible subtle changes in the ANS, the different clinical evaluation methods, the differences in the sample size, and the individual differences in the onset and progression of the disease could explain the discrepancies between the results.

The dynamics of blood pressure and heart rate, particularly the ability to restore homeostasis after standing, depend largely on the state of ANS [43]. Interestingly, our results do not identify orthostatic hypotension as a significant factor for falls. These results are important evidence that HRV is an independent cardiac factor isolated from changes in blood pressure during postural changes, at least in the population with HD at early and middle stages, as previously has been reported [12].

Little is known about possible alterations of vascular sympathetic regulatory activity in patients without orthostatic hypotension or symptoms of orthostatic intolerance in other neurodegenerative diseases [44]. While not well documented in HD patients, orthostatic hypotension has been extensively examined in Parkinson's disease to whom about 50\% without hypotension, a certain degree of sympathetic cardiac abnormalities could be identified in the supine position [45]. In this sense, we would have to weigh some aspects of the analysis of this dissociation. Fist, the exact origin of the damage of the ANS function in individuals with HD has not yet been elucidated $[10,15]$; second, it has been postulated that also HDrelated cardiac alterations are likely driven by CNS dysfunctions [46-47], and third, there is no reason to suspect damage to the peripheral nervous system $[10,15]$ nor vascular dysfunction in animal models [46]. Then, it is possible to assume that, as in Parkinson's disease, the initial impairment of the sympathetic vasomotor control could mainly affect the variability of blood pressure, and only subsequently induce 
changes in the mean values of systolic blood pressure that cause orthostatic hypotension? [44]. In this respect, other mechanisms may explain the blood pressure values maintained during standing position in Parkinson's disease, including a noradrenergic hypersensitivity [44]. Unfortunately, blood pressure variability and changes in catecholamines during gravitational stimulation were not addressed in the present study. Therefore we hypothesized that the dissociation between HRV and orthostatic phenomena could not be so surprising in the early-mid stages of HD. This allows us to postulate the hypothesis that the autonomic changes registered with the ECG are probably more sensitive to predict RoF than those dependent on orthostatic hypotension. However, additional research should test this hypothesis

A relevant result of the study was the demonstration of the association between the decreases in parasympathetic prevalence measured by HRV and be a recurrent faller independently of orthostatic phenomena. A possible explanation of the association would be a state of reactivity (hyperarousal), due to a well-determined early hyper-sympathetic state in HD [15-17], which in turn would be associated with having a greater awareness of the motor disability in tasks that challenge balance and potentially translate as fear of falling (FoF) [48]. Within this framework, another hypothesis states that the activity of the autonomic nervous system is a major component of the emotional response to stress, with large differences ranging from undifferentiated excitation to the recognition of a highly specific response for certain stressful emotions such as the fear [49]. In line with our results, experimental models have demonstrated that different fearful events rapidly increased heart rate (HR), decrease the parasympathetic coupled with uninhibited sympathetic activities, and indexed by low HRV [50-51]. Future studies should be better to explain the proposed mechanisms.

Contrary to our expectations, we found that the number of self-reported falls in the previous 12 -months did not correlate with any balance variables recommended measuring the RoF in HD (BBS, TUG, and TMT) [23] (data not shown). We hypothesize that this could potentially be related to two reasons. First, methodologically, it is difficult to obtain accurate falls data from people with HD due to recall bias, cognitive deficits, and behavioral issues that may impact on the accuracy of retrospective data $[2,52]$ Second, the RoF assessment is used to measure and classify patients at high or low RoF, unfortunately administrating functional test may, therefore, be affected by responder-bias and inter-observer variability. A few studies have found the TUG test $[4,23,25]$, the BBS [4, 22-23] and the TMT [23, 26-27], with cutoffs of $\geq 14$ seconds, 40 points or less and $\leq 21$ or less respectively, have been associated with increased fall risk in HD. These data demonstrate that simple physical outcome measures cannot 
accurately predict falls, and other intrinsic and extrinsic factors should be considered. In this regard, after the logistic regression analysis, our results showed that two indexes of HRV (RMSSD-resting and LF / HF ratio in standing state) were better than the three most commonly used scales to measure RoF in HD, in the prediction of odds to be a recurrent faller in the next six-months (e.g. almost fivefold times the odds to be a recurrent faller if higher LH/HF ratio in standing state is registered).

The strengths of this study were the use of an easy and useful tool to measure short-term HRV through postural changes; the use of an extensive balance, gait and neuropsychological testing battery that captures multiple possible risk factors and their potential correlations with fall risk; and novel description of qualitative aspects of falls in HD.

We acknowledge that this is an exploratory study. Shortcomings that could affect the accuracy of our conclusions should be mentioned. First, the small sample size and the clinical heterogeneity of our participants must be considered. Second, unfortunately, and due to the exploratory nature of the study, neither baroreflex sensitivity (BRS) nor any dynamic interaction index of cardiac period systolic blood pressure was available for baroreflex control analysis during the postural challenge. These indices would have characterized the cardiac baroreflex from the spontaneous fluctuations of the cardiac period and the systolic blood pressure (HP-SAP) when facing the orthostatic challenge and variations in the blood pressure buffer [53]. Third, the results of the logistic regression analysis must be interpreted with caution due to the small sample size. Although binary logistic regression recommendations propose a minimum of 5-9 events per variable (EPV) [54-55], the current sample size might not avoid type I or II errors. Fourth, the obtained results of HRV analysis should be interpreted with caution since the effect of the consumption of caffeine-containing beverages is usually longer than the three hours recommended in the study. Hence, these results should be corroborated by cross-validated further investigations, using larger samples across different stages of the disease and a longer prospective follow-up.

In conclusion, our findings suggest that an increased sympathetic prevalence, as detected by HRV measures, is associated with the odds of being a recurrent faller in early-mid stages of HD population, independent of orthostatic phenomena. Future studies should address whether HRV measures may represent a predictive parameter for RoF in the early-mid stages of HD and whether such association persists in more advanced stages of the disease. Inclusion of short registry of HRV in the early assessment 
of HD patients may be relevant to early detection and reduce the recurrent falls as well to identify and monitor those patients at high RoF who would benefit from fall prevention programs.

\section{BIBLIOGRAPHY}

1. Jernigan TL, Salmon DP, Butters N, Hesselink JR. (1991). Cerebral Structure on MRI, Part II: Specific Changes in Alzheimer's and Huntington's Diseases. Biol Psychiatry. 29(1):68-81.

2. Vuong K, Canning CG, Menant JC, Loy CT. (2018). Gait, balance, and falls in Huntington disease.

Handbook of Clinical Neurology. Elsevier B.V. 1st Ed. Vol. 159. 251-260 p.

3. Grimbergen YAM, Knol MJ, Bloem BR, Kremer BPH, Roos RAC, Munneke M. (2008). Falls and Gait Disturbances in Huntington 's Disease. Mov Disord. 23(7):970-976.

4. Busse ME, Khalil H, Quinn L, Rosser AE. (2008). Physical Therapy Intervention for People with Huntington Disease. Phys Ther. 88(7):820-831.

5. Wheelock VL, Tempkin T, Marder K, Nance M, Myers RHP, Zhao H, et al. (2003). Predictors of Nursing Home Placement in Huntington Disease: Neurology. 60(6):998-1001.

6. Nocua R, Noury N, Gehin C, Dittmar A, McAdams E. (2009). Evaluation of the autonomic nervous system for fall detection. In: Proceedings of the 31st Annual International Conference of the IEEE Engineering in Medicine and Biology Society: Engineering the Future of Biomedicine, EMBC 2009.

7. Stolze H, Klebe S, Zechlin C, Baecker C, Friege L, Deuschl G. (2004). Falls in frequent neurological diseases: Prevalence, risk factors and aetiology. J Neurol. 251(1):79-84.

8. Melillo P, Castaldo R, Izzo R, De Luca N, Pecchia L. (2017). Fall Prediction in Hypertensive Patients via Short-Term HRV Analysis. IEEE J Biomed Health Inform. 21(2):399-406.

9. Razjouyan J, Grewal GS, Rishel C, Parthasarathy S, Mohler J NB. (2012). Activity Monitoring and Heart Rate Variability as Indicators of Fall Risk. J Gerontol Nurs. 43(7):1-10.

10. Andrich J, Schmitz T, Saft C, Postert T, Kraus P, Epplen JT, et al. (2002). Autonomic nervous system function in Huntington's disease. J Neurol Neurosurg Psychiatry. 72(6):726-731.

11. Sharma K. (2002). Sympathetic skin response and the heart rate variability in patients with Huntington's disease. Electroencephalogr Clin Neurophysiol. 103(1):138. 
12. Bär KJ, Boettger MK, Andrich J, Epplen JT, Fischer F, Cordes J, et al. (2008). Cardiovagal modulation upon postural change is altered in Huntington's disease. Eur J Neurol. 15(8):869-871.

13. Cankar K, Melik Z, Kobal J, Starc V. (2018). Evidence of cardiac electrical remodeling in patients with Huntington disease. Brain Behav. 8(8):1-9.

14. Bellosta Diago E, Pérez Pérez J, Santos Lasaosa S, Viloria Alebesque A, Martínez Horta S, Kulisevsky J, et al. (2017). Circadian rhythm and autonomic dysfunction in presymptomatic and early Huntington's disease. Park Relat Disord. 44:95-100.

15. Kobal J, Meglič B, Mesec A, Peterlin B. (2004). Early sympathetic hyperactivity in Huntington’s disease. Eur J Neurol. 11(12):842-848.

16. Bellosta Diago E, Pérez-Pérez J, Santos Lasaosa S, Viloria Alebesque A, Martínez-Horta S, Kulisevsky J, et al. (2018). Neurocardiovascular pathology in pre-manifest and early-stage Huntington's disease. Eur J Neurol. 25(7):956-962.

17. Benarroch EE. (1993). The Central Autonomic Network: Functional Organization, Dysfunction, and Perspective. Mayo Clin Proc. 68(10):988-1001.

18. Rosas HD, Salas DH, Lee SY, Zaleta AK, Pappu V, Fischl B, Greve D, Hevelone N HS. (2008). Cerebral cortex and the cinical expression of Huntington's disease: complexity and heterogeneity. Brain. 131(4):1057-1068.

19. Hegde R, Sudarshan B, Kumar SCP, Hariprasad S, Satyanarayana B. (2013). Technical advances in fall detection system - a review. Int J Comput Sci Mob Comput. 2:152-160.

20. Boyé NDA, Mattaceraso FUS, Lieshout EMM Van, Hartholt KA. (2015). Physical performance and quality of life in single and recurrent fallers : Data from the Improving Medication Prescribing to Reduce Risk of Falls study. Geriatr Gerontol Int. 15:350-355.

21. Gibson M, Andres R, Isaacs B W-PJ. (1987). The prevention of falls in later life. A report of the Kellogg International Work Group on the Prevention of Falls by the Elderly. Dan Med Bull. 34(S4):1-24.

22. Berg KO, Wood-Dauphinee SL, Williams JI MB. (1992). Measuring balance in the elderly: validation of an instrument. Can J Public Health. 83(2):S7-91. 
23. Mestre TA. (2018). Rating Scales and Performance-based Measures for Assessment of Functional Ability in Huntington's Disease : Critique and Recommendations Performance-based. Mov Disord Clin Pract. 5(4):361-372.

24. Podsiadlo D, Richardson S. (1991). The Timed "Up \&Go": A Test of Basic Functional Mobility for Frail Elderly Persons. J Am Geriatr Soc. 39:142-148.

25. Quinn L, Khalil H, Dawes H, Fritz NE, Kegelmeyer D, Kloos AD, et al. (2013). Reliability and Minimal Detectable Change of Physical Performance Measures in Individuals With Pre-manifest and Manifest Huntington Disease. Phys Ther. 93(7):942-956.

26. Tinetti ME. (1986). Performance-Oriented Assessment of Mobility Problems in Elderly Patients. J Am Geriatr Soc. 34:119-126.

27. Kloos AD, Kegelmeyer DA, Young GS, Kostyk SK. (2010). Fall Risk Assessment Using the Tinetti Mobility Test in Individuals with Huntington’s Disease. 25(16):2838-2844.

28. Lamb SE, Jørstad-Stein EC, Hauer K, Becker C. (2005). Development of a common outcome data set for fall injury prevention trials: The Prevention of Falls Network Europe consensus. J Am Geriatr Soc. 53(9):1618-1622.

29. Freeman R, Wieling W, Axelrod FB, Benditt DG, Benarroch E. (2011). Consensus statement on the definition of orthostatic hypotension, neurally mediated syncope and the postural tachycardia syndrome.

Clin Auton Res. 21:69-72.

30. Tarvainen, Mika P. Ranta-aho PO, Karjalainen PA, Lipponen JA, van Iersel MB. (2013). Kubios HRV - Heart rate variability analysis software. Comput Methods Programs Biomed. 113(1):210-220.

31. Ho KK, Moody GB, Peng CK, Mietus JE, Larson MG, et al. (1997). Predicting survival in heart failure case and control subjects by use of fully automated methods for deriving nonlinear and conventional indices of heart rate dynamics. Circulation. 96: 842-848.

32. Heart rate variability. Standards of measurement, physiological interpretation, and clinical use. (1996).Task Force of The European Society of Cardiology and The North American Society of Pacing and Electrophysiology. Eur Heart J. 17:354-381. 
33. Tarkiainen TH, Kuusela TA, Tahvanainen KUO, Hartikainen JEK, Tiittanen P, Timonen KL, et al. (2007). Comparison of methods for editing of ectopic beats in measurements of short-term non-linear heart rate dynamics. Clin Physiol Funct Imaging. 27(2):126-133.

34. Seely A, T Macklem P. Seeley AJE, Macklem PT. (2005). Complex systems and the technology of variability analysis. Crit Care 8:R367-R384. Crit Care. 8(6):R367-384.

35. Iyengar N, Peng CK, Morin R, Goldberger AL, Lipsitz LA. (1996). Age-related alterations in the fractal scaling of cardiac interbeat interval dynamics. Am J Physiol. 271(4 Pt 2):R1078-1084.

36. Richman JS, Moorman JR. (2017). Physiological time-series analysis using approximate entropy and sample entropy. Am J Physiol Circ Physiol. 278(6):H2039-2049.

37. Vigo DE, Dominguez J, Guinjoan SM, Scaramal M, Ruffa E, Solernó J, et al. (2010). Nonlinear analysis of heart rate variability within independent frequency components during the sleep-wake cycle. Auton Neurosci Basic Clin. 154(1-2):84-88.

38. Hayano J and Yuda E. (2019). Pitfalls of assessment of autonomic function by heart rate variability J Physiol Anthrop. 38:3

39. Bird ED. (1997). Neuroendocrine changes in Huntington's disease. Adv Neurol. 23:291-297.

40. Melik Z, Kobal J, Cankar K, Strucl M. (2012). Microcirculation response to local cooling in patients with Huntington's disease. J Neurol. 259(5):921-928.

41. Kobal J, Melik Z, Cankar K, Bajrovic FF, Meglic B, Peterlin B, et al. (2010). Autonomic dysfunction in presymptomatic and early symptomatic Huntington's disease. Acta Neurol Scand. 121(6):392-399.

42. Sannino G, Melillo P, Stranges S, De Pietro G, Pecchia L. (2015). Short term Heart Rate Variability to predict blood pressure drops due to standing: A pilot study. BMC Med Inform Decis Mak. 15(3):S2. 43. Sannino G, Melillo P, De Pietro G, Stranges S, Pecchia L. (2014). To What Extent It Is Possible to Predict Falls due to Standing Hypotension by Using HRV and Wearable Devices? Study Design and Preliminary Results from a Proof-of-Concept Study. Ambient Assist Living Dly Act Springer Int Publ. $167-170$.

44. Barbic F, Perego F, Canesi M, et al. (2007). Early abnormalities of vascular and cardiac autonomic 
control in Parkinson's disease without orthostatic hypotension. Hypertension. 49(1):120-126.

45. Goldstein DS, Holmes CS, Dendi R, et al. (2002). Orthostatic hypotension from sympathetic denervation in Parkinson's disease. Neurology. 58:1247-1255.

46. Di Pardo A, Carrizo A, Damato A, et al. (2017). Motor phenotype is not associated with vascular dysfunction in symptomatic Huntington's disease transgenic R6/2 (160 CAG) mice. Sci Rep. 17;7: 42797.

47. Park S \& Colwell S. (2019). Do Disruptions in the Circadian Timing System Contribute to Autonomic Dysfunction in Huntington's disease? Yale J Biol Med. 92(2): 291-303.

48. Young WR, Williams AM (2015). How fear of falling can increase the risk of falls in older adults: applying psychological theory to practical observations. Gait posture. 41 (1): 7-12.

49. Kreibig SD. Autonomic nervous system activity in emotion: A review (2010). Biological Psychology. 84: 394-421.

50. Kim HG, Cheon EJ, Bai DS, Lee YH, Koo BH (2018). Stress and heart rate variability: A metaanalysis and review of the literature. 15(3):235-245.

51. Wu R, Gu R, Yang K, Luo YJ (2019). How Do Amusement, Anger and Fear Influence Heart Rate and Heart Rate Variability? Front Neurosci. 18 (13):1131

52. Purcell NL, Goldman JG, Ouyang B, Bernard B, O’Keefe JA. (2019). The Effects of Dual-Task Cognitive Interference and Environmental Challenges on Balance in Huntington's disease. Mov Disord Clin Pract. 6(3):202-212.

53. Milan-Mattos JC, Porta A, Perseguini NM, et al. (2018). Influence of age and gender on the phase and strength of the relation between heart period and systolic blood pressure spontaneous fluctuations. J Appl Physiol. 124 (3):791-804.

54. Vittinghof E \& McCulloch CE. (2006). Relaxing the rule of ten events per variable in logistic and Cox regression. Am J Epidemiol. 165:710-718.

55. Pavlou M, Ambler G, Seaman SR. Guttmann O, Elliott P, et al. (2015). How to develop a more accurate risk prediction model when there are few events. BMJ. 351: h3868. 
Table 1. Clinical differences between single and recurrent fallers at baseline.

\begin{tabular}{|c|c|c|c|c|}
\hline & $\begin{array}{l}\text { Total } \\
(\mathbf{n}=\mathbf{2 0})\end{array}$ & $\begin{array}{l}\text { Single } \\
\text { fallers } \\
(\mathrm{n}=7)\end{array}$ & $\begin{array}{l}\text { Recurrent } \\
\text { fallers } \\
(n=13)\end{array}$ & $p$ value ${ }^{*}$ \\
\hline Age, years ${ }^{\mathrm{a}}$ & $55(44-66)$ & $51(33-73)$ & $57(44-65)$ & 0.699 \\
\hline Female gender, n (\%) & $12(60)$ & $3(43)$ & $9(69)$ & 0.251 \\
\hline Illness duration ${ }^{a}$ & $4.5(3-7)$ & $3(0-5)$ & $5(4-8)$ & 0.115 \\
\hline CAG-repeat length ${ }^{\mathrm{a}}$ & $42(40-46)$ & $42(40-46)$ & $42(40-46)$ & 0.938 \\
\hline Weight $(\mathbf{k g})^{\mathrm{a}}$ & $66.6(58.8-76.5)$ & $70(48-90)$ & $64.7(59-73)$ & 0.643 \\
\hline $\mathrm{MoCA}^{\mathrm{a}}$ & $22(19-25)$ & $25(17-26)$ & $22(21-23)$ & 0.699 \\
\hline $\mathbf{F A B}^{\mathrm{a}}$ & $13(10-16)$ & $13(8-18)$ & $13(11-15)$ & 0.877 \\
\hline UHDRS-TMS $^{\mathrm{a}}$ & $34.5(28-49)$ & $32(5-53)$ & $39(33-48)$ & 0.393 \\
\hline UHDRS-TFC $^{\mathbf{a}}$ & $11(7.5-12.5)$ & $13(11-13)$ & $11(7-12)$ & 0.081 \\
\hline \multicolumn{5}{|l|}{ Stages of disease } \\
\hline Early-mid, n (\%) & $16(80)$ & $6(86)$ & $10(77)$ & \multirow{2}{*}{0.260} \\
\hline Late, n (\%) & $4(20)$ & $1(14)$ & $3(23)$ & \\
\hline Falls number ${ }^{a}$ & $2(1-4)$ & $0(0-1)$ & $3(2-4)$ & $<0.001$ \\
\hline FES-I ${ }^{\text {a }}$ & $19(16.5-29)$ & $17(16-19)$ & $22(18-30)$ & 0.043 \\
\hline BBS score ${ }^{a}$ & $50(42-52.5)$ & $52(42-56)$ & $50(42-51)$ & 0.275 \\
\hline TUG seconds & $14.2(9.7-17.9)$ & $15.4(10.4-22.7)$ & $14.1(9-16.7)$ & 0.588 \\
\hline TMT score ${ }^{a}$ & $21.5(15.5-24)$ & $24(13-26)$ & $21(16-23)$ & 0.393 \\
\hline SAS score ${ }^{a}$ & $15(5.5-27.5)$ & $6(4-28)$ & $16(13-21)$ & 0.588 \\
\hline BDI $^{\mathbf{a}}$ & $11(3.5-18)$ & $4(3-14)$ & $14(8-21)$ & 0.097 \\
\hline $\mathbf{B A I}^{\mathbf{a}}$ & $7.5(4-10.5)$ & $4(3-8)$ & $9(7-13)$ & 0.067 \\
\hline Barthel Index ${ }^{a}$ & $95(77.5-100)$ & $100(80-100)$ & $90(70-100)$ & 0.211 \\
\hline \multicolumn{5}{|l|}{ High risk of falls } \\
\hline BBS, n (\%) & $7(35)$ & $2(29)$ & $5(39)$ & 0.658 \\
\hline TUG, n (\%) & $14(70)$ & $5(71)$ & $9(69)$ & 0.919 \\
\hline TMT, n (\%) & $10(50)$ & $3(43)$ & $7(54)$ & 0.639 \\
\hline
\end{tabular}

*Significant at $\mathrm{p}<0.05$. Significant comparison Mann Whitney $\mathrm{U}$ test or $\mathrm{X}^{2}$ test $(\mathrm{df}=1.20)$ are highlighted in bold.

(a) Data are expressed as median (interquartile range 25\%-75\%) or $\mathrm{n}(\%)$ as frequency (percentage of columns). Abbreviations: MoCA: Montreal Cognitive Assessment; FAB: Frontal Assessment Battery; UHDRS-TMS: Unified Huntington's disease rating scale Total motor score; UHDRS-TFC: Unified Huntington's disease rating scale Total functional capacity; BBS: Berg Balance Scale; TUG: Timed-up go test; TMT: Tinetti Mobility Test; SAS: Starkstein's Apathy scale; BDI: Beck Depression Index; BAI: Beck Anxiety Index; FES-I: International Fear of falling

Scale. 
Table 2. Association between falls and HRV parameters during postural changes at baseline (single fallers $n=7$, recurrent fallers $n=13$ ).

\begin{tabular}{|c|c|c|c|c|c|c|c|c|c|}
\hline & \multicolumn{3}{|l|}{ Resting-State } & \multicolumn{3}{|l|}{ Standing State } & \multicolumn{3}{|c|}{ Differences between states } \\
\hline & $\begin{array}{l}\text { Single } \\
\text { fallers }\end{array}$ & $\begin{array}{l}\text { Recurrent } \\
\text { fallers }\end{array}$ & $\begin{array}{l}p \\
\text { value* }\end{array}$ & $\begin{array}{l}\text { Single } \\
\text { fallers }\end{array}$ & $\begin{array}{l}\text { Recurrent } \\
\text { fallers }\end{array}$ & $\begin{array}{l}p \\
\text { value* }\end{array}$ & $\begin{array}{l}\text { Single } \\
\text { fallers }\end{array}$ & $\begin{array}{l}\text { Recurrent } \\
\text { fallers }\end{array}$ & $\begin{array}{l}p \\
\text { value* }^{*}\end{array}$ \\
\hline HRM (bpm) & $77.2(59.3-87.3)$ & $77.4(67-86.6)$ & 0.877 & $89(65.9-94.8)$ & $78.4(73.3-88.8)$ & 0.817 & $7.3(4.8-10.4)$ & $4.9(2.6-7.2)$ & 0.275 \\
\hline RRM (ms) & $777.1(687-1011.1)$ & $774.8(692.7-895.1)$ & 0.877 & $674(633-910.9)$ & $765.6(675.6-818.9)$ & 0.817 & $83.6(54-103.1)$ & $44.2(29.4-93.7)$ & 0.211 \\
\hline SDNN (ms) & $64.8(41.1-68.6)$ & $43.8(32.8-64.3)$ & 0.351 & $58.9(46-106.1)$ & $42.1(35.2-57)$ & 0.115 & $-2.9(-26.2-2.8)$ & $2.2(-9.3-4.7)$ & 0.393 \\
\hline RMSSD (ms) & $52.7(39.3-73.2)$ & $26.3(19.6-46.1)$ & 0.011 & $55.7(37.3-67.1)$ & $22.7(17.7-53.3)$ & 0.081 & $5(-5.6-8.9)$ & $2.8(-10.1-3.8)$ & 0.211 \\
\hline $\ln \mathrm{VLF}\left(\mathrm{ms}^{2}\right)$ & $7.4(5-7.7)$ & $6.6(5.6-7.3)$ & 0.393 & $6.8(5.5-8.2)$ & $6.5(6.1-7.1)$ & 0.757 & $-0.3(-0.5-0.6)$ & $-0.3(-0.7-0.5)$ & 0.938 \\
\hline $\ln \mathrm{LF}\left(\mathrm{ms}^{2}\right)$ & $6.9(5.1-7.2)$ & $6.5(6-7.5)$ & 0.877 & $5.4(5.1-7.1)$ & $6.5(5.7-7.4)$ & 0.485 & $0.1(-0.5-0.6)$ & $0.1(-0.3-0.5)$ & 0.877 \\
\hline $\ln \mathrm{HF}\left(\mathrm{ms}^{2}\right)$ & $7.3(4.9-7.4)$ & $5.4(4.5-5.7)$ & 0.081 & $6.9(5.2-7.1)$ & $5.2(4.3-6.3)$ & 0.157 & $0.3(-0.2-1)$ & $0.1(-0.5-0.3)$ & 0.241 \\
\hline $\mathrm{LF} / \mathrm{HF}$ & $0.9(0.6-1.2)$ & $2.6(1.9-4)$ & 0.006 & $0.9(0.4-1.3)$ & $2.7(1-3.2)$ & 0.019 & $0.2(-0.1-0.5)$ & $0.2(-0.1-1.6)$ & 0.643 \\
\hline DFA- $\alpha 1$ & $0.9(0.7-1)$ & $1.2(1-1.4)$ & 0.024 & $0.9(0.7-0.9)$ & $1.3(1.2-1.4)$ & 0.006 & $0.04(-0.3-0.2)$ & $-0.03(-0.2-0.1)$ & 0.699 \\
\hline SampEn & $1.2(0.9-1.9)$ & $1.3(1.1-1.5)$ & 0.643 & $1.2(0.9-1.3)$ & $1.2(1-1.4)$ & 0.183 & $0.3(0.1-0.5)$ & $0.1(-0.1-0.2)$ & 0.067 \\
\hline
\end{tabular}

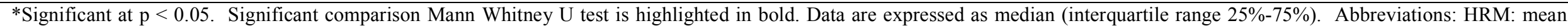

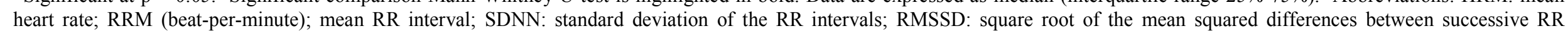

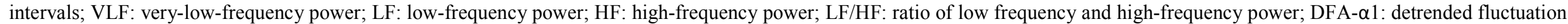
analysis short term parameter; SampEn: sample entropy. 
Table 3. Association between falls and HRV parameters during postural changes at six-month follow-up (single fallers $n=11$, recurrent fallers $n=7$ ).

\begin{tabular}{|c|c|c|c|c|c|c|c|c|c|}
\hline & \multicolumn{3}{|l|}{ Resting State } & \multicolumn{3}{|l|}{ Standing State } & \multicolumn{3}{|c|}{ Differences between states } \\
\hline & $\begin{array}{l}\text { Single } \\
\text { fallers }\end{array}$ & $\begin{array}{l}\text { Recurrent } \\
\text { fallers }\end{array}$ & $p$ value* & $\begin{array}{l}\text { Single } \\
\text { fallers }\end{array}$ & $\begin{array}{l}\text { Recurrent } \\
\text { fallers }\end{array}$ & $\begin{array}{l}p \\
\text { value* }\end{array}$ & $\begin{array}{l}\text { Single } \\
\text { fallers }\end{array}$ & $\begin{array}{l}\text { Recurrent } \\
\text { fallers }\end{array}$ & $\begin{array}{l}p \\
\text { value* }\end{array}$ \\
\hline HRM (bpm) & $71.1(59.3-84.5)$ & $79.1(56.8-95.5)$ & 0.596 & $74.9(65.9-91.7)$ & $79.8(63.6-94.9)$ & 1.000 & $6.5(4.3-7.8)$ & $4.9(-4.2-7.2)$ & 0.328 \\
\hline RRM (ms) & $843.9(710-1011.1)$ & $774.8(828-1055.5)$ & 0.596 & $801.4(654.2-910.9)$ & $708.6(632.3-944.1)$ & 1.000 & $83.6(34.4-103.1)$ & $60.4(-44.1-111.4)$ & 0.285 \\
\hline SDNN (ms) & $64.3(41.8-68.6)$ & $43.8(24.8-74.7)$ & 0.246 & $57(45.4-101.3)$ & $42.1(24-132.8)$ & 0.179 & $1.2(-26.2-4.2)$ & $0.8(-31.3-4.7)$ & 0.596 \\
\hline $\operatorname{RMSSD}(\mathrm{ms})$ & $46.1(31.7-59.5)$ & $25.3(19.4-41.8)$ & 0.027 & $55.7(30.4-67.1)$ & $18.6(15.6-45.3)$ & 0.069 & $4.1(-14.6-6.2)$ & $3.3(-3.5-3.8)$ & 0.479 \\
\hline $\ln \operatorname{VLF}\left(\mathrm{ms}^{2)}\right.$ & $7.3(6.6-7.7)$ & $6.4(5.6-7.3)$ & 0.211 & $6.8(5.7-7.7)$ & $7(6.3-9.1)$ & 0.930 & $0.3(-0.5-0.9)$ & $-0.4(-0.7-0.1)$ & 0.285 \\
\hline $\ln \mathrm{LF}\left(\mathrm{ms}^{2}\right)$ & $6.8(5.7-7.2)$ & $6.5(4.9-7.6)$ & 0.791 & $5.8(5.2-7.2)$ & $6.9(4.8-7.8)$ & 0.930 & $0.1(-0.5-0.6)$ & $0.1(-0.2-0.5)$ & 0.596 \\
\hline $\ln \mathrm{HF}\left(\mathrm{ms}^{2}\right)$ & $7.1(4.9-7.4)$ & $5.3(4.3-6.1)$ & 0.069 & $6.6(5.1-7.1)$ & $5.5(3.8-7.7)$ & 0.085 & $0.3(-0.3-0.5)$ & $0.1(-0.5-0.5)$ & 1.00 \\
\hline $\mathrm{LF} / \mathrm{HF}$ & $1.2(0.9-2.1)$ & $2.6(1.2-7.6)$ & 0.085 & $0.9(0.8-1.4)$ & $3.2(0.9-5.7)$ & 0.035 & $0.2(-0.1-1.3)$ & $0.3(-0.6-1.9)$ & 0.860 \\
\hline DFA- $\alpha 1$ & $1(0.7-1.4)$ & $1.2(0.8-1.5)$ & 0.375 & $0.9(0.7-1.2)$ & $1.3(0.7-1.5)$ & 0.085 & $0.04(-0.2-0.2)$ & $-0.03(-0.2-0.4)$ & 0.425 \\
\hline SampEn & $1.2(0.9-1.6)$ & $1.3(1.1-1.6)$ & 0.536 & $1.2(0.9-1.3)$ & $1.3(0.7-1.4)$ & 0.425 & $0.1(-0.03-0.4)$ & $0.1(-0.1-0.2)$ & 0.211 \\
\hline
\end{tabular}

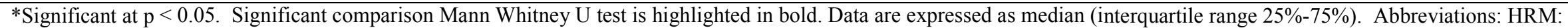

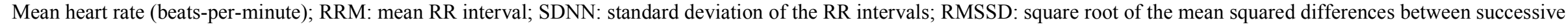

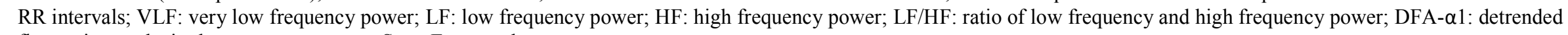
fluctuation analysis short-term parameter; SampEn: sample entropy. 

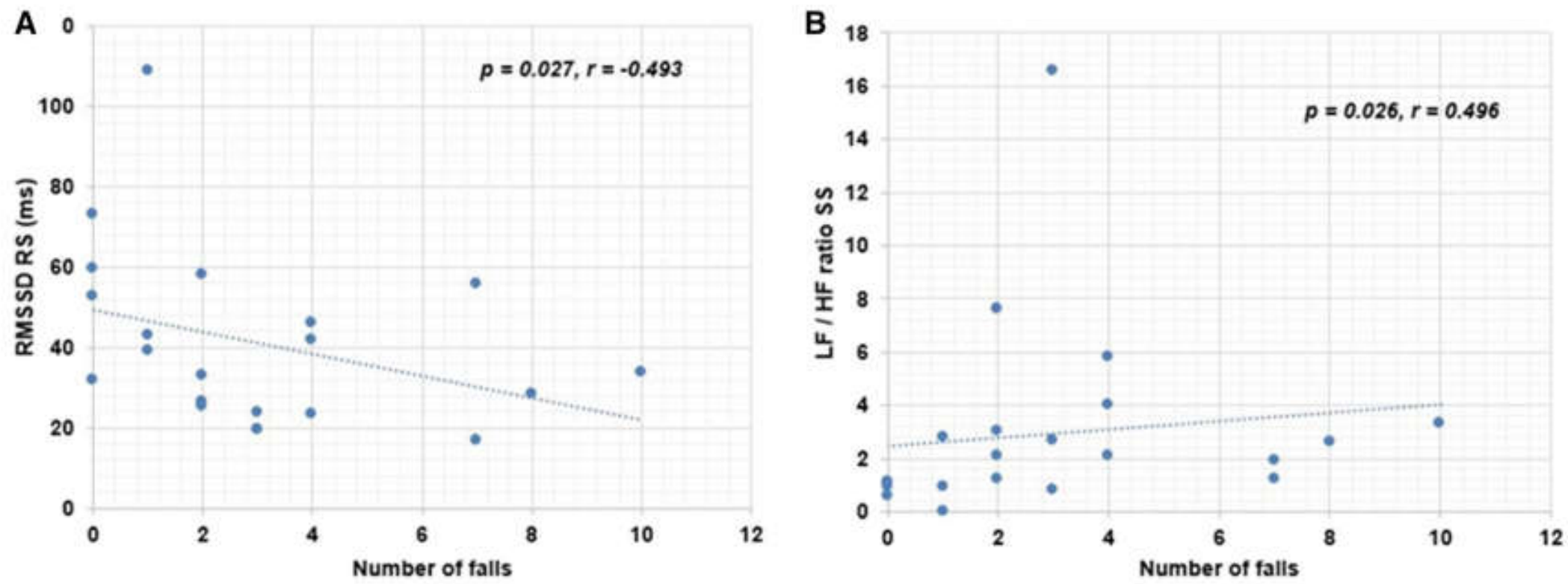

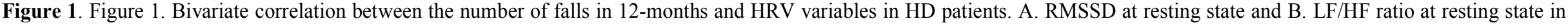
$\mathrm{HD}$ patients. RMSSD = square root of the mean squared differences between successive RR intervals (ms). LF/HF $=$ ratio of low frequency and high-frequency power. 